\title{
Breast Health
}

\author{
Sinisa Franjic* \\ Independent Researcher, Herzegovina \\ Submission: May 21, 2021; Published: June 02, 2021 \\ *Corresponding author: Siniša Franjić, Independent Researcher, Herzegovina
}

\begin{abstract}
Breasts are subject to change. They react to hormonal changes, they can hurt, and they also react to food, lifestyle and stress. Breast health is very important and self-examination should not be missed. By palpation and self-examination of the breasts, which are heterogeneous due to cyclic exposure to hormones, it is impossible to make any diagnosis. However, self-examination of women is extremely important after menstruation when there are less physiological "bumps" than during the cycle. It is very important that women know their breasts well so that they can notice when something new appears in them. Depending on age and anamnesis, it is extremely important to go for a mammogram and compare images and perform comparative ultrasound examinations. Any new change should be discussed with doctor
\end{abstract}

Keywords: Breasts; Abnormalities; Pain; Cancer; Health

\section{Introduction}

Breast conditions are common in women [1]. Estimates are that $90 \%$ of women show benign breast tissue changes when examined microscopically. The ultimate goal is to empower women to take control of their breast health by knowing what their breasts look and feel like and to use fundamental health and wellness principles such as diet and exercise to create a healthy environment in the body. Women who regularly perform breast self-exams may note changes in the breasts during the menstrual cycle. The breasts become more lumpy and tender as menses approaches and less lumpy and less tender after menses.

\section{Structure}

The female breasts are each composed of about twenty lobes of glandular tissue embedded in fibrous and adipose tissue [2]. The lobes are arranged circumferentially much as petals on a flower. Each lobe consists of clusters of glands (terminal ductules or acini) in which milk is made during pregnancy, and a series of intralobular ducts connecting the acini and the stromal tissue in which the acini and ducts are located. Taken together these three components are called the terminal duct lobular unit (TDLU). The ducts of the TDLU converge to form large ducts that extend to the nipple. The ductules and ducts of the lobular system are bilayered. The luminal epithelial cells lining the ducts are the source of milk during lactation and also of much breast pathology, the most important being carcinoma. The outer layer is myoepithelial in nature and characteristically is lost in invasive breast cancer. Preservation versus loss of the bilayered nature of the lobular system is an important diagnostic criterion in separating benign from malignant breast disease.

The breasts are modified sweat glands that have become specialized to secrete milk. Before puberty, breast tissue in both sexes consists only of branching ducts and fibrous tissue without glandular tissue or fat. In the female, the breasts enlarge at puberty in response to estrogen and progesterone produced by the ovaries, whereas the unstimulated male breasts retain their prepubertal form. Postpuber Independent Researcher, Herzegovinatal changes in the female include proliferation of glandular and fibrous tissue and accumulation of adipose tissue within the breasts. Variations in the size of the postpubertal breasts of nonpregnant women are primarily the result of variations in the amount of fat and fibrous tissue in the breasts rather than differences in the amount of glandular tissue.

\section{Changes}

Once menses is established, the breast undergoes a periodic premenstrual phase during which the acinar cells increase in number and size, the ductal lumens widen, and breast size and turgor increase slightly [3]. Many women have breast tenderness during this phase of the menstrual cycle. Menstrual bleeding is followed by a postmenstrual phase, characterized by a decrease in size and turgor, reduction in the number and size of the breast acini, and a decrease in diameter of the lactiferous ducts. Cyclic hormonal influences to the breast are quite variable. 
In response to progesterone during pregnancy, breast size and turgidity increase considerably. These changes are accompanied by deepening pigmentation of the nipple-areolar complex, nipple enlargement, areolar widening, and an increase in the number and size of the lubricating glands in the areola. The breast ductal system branches markedly, and the individual ducts widen. The acini increase in number and size. In late pregnancy, the fatty tissues of the breasts are almost completely replaced by cellular breast parenchyma. After delivery with the rapid drop in progesterone and estrogen levels, the breasts, now fully mature, start to secrete milk. With cessation of nursing or administration of estrogens, which inhibit lactation, the breast rapidly returns to its pre pregnancy state, with marked diminution of cellular elements and an increase in adipose deposits.

Following menopause, which typically occurs during the fifth decade of life, the breast undergoes a gradual process of atrophy and involution. There is a decrease in the number and size of acinar and ductal elements, so that the breast tissue regresses to an almost infantile state. Adipose tissue may or may not atrophy, with disappearance of the parenchymal elements.

\section{Breasts}

The two mammary glands, or breasts, are accessory organs of the female reproductive system that are specialized to secrete milk following pregnancy [4]. They overlie the pectoralis major muscles and extend from the second to the sixth ribs and from the sternum to the axilla. Each breast has a nipple located near the tip, which is surrounded by a circular area of pigmented skin called the areola. Each breast is composed of approximately 9 lobes (the number can range between 4 and 18), which contain glands (alveolar) and a duct (lactiferous) that leads to the nipple and opens to the outside. The lobes are separated by dense connective and adipose tissues, which also help support the weight of the breasts. During pregnancy, placental estrogen and progesterone stimulate the development of the mammary glands. Because of this hormonal activity, the breasts may double in size during pregnancy. At the same time, glandular tissue replaces the adipose tissue of the breasts.

Following childbirth and the expulsion of the placenta, levels of placental hormones (progesterone and lactogen) fall rapidly, and the action of prolactin (milk-producing hormone) is no longer inhibited. Prolactin stimulates the production of milk within a few days after childbirth, but in the interim, a dark yellow fluid called colostrum is secreted. Colostrum contains more minerals and protein, but less sugar and fat, than mature breast milk. Colostrum secretion may continue for approximately a week after childbirth, with gradual conversion to mature milk. Colostrum is rich in maternal antibodies, especially immunoglobulin A (IgA), which offers protection for the newborn against enteric pathogens.

\section{Breast Milk}

Production of human breast milk among healthy mothers who deliver full-term infants occurs in three phases-colostrum, transitional milk, and mature milk [5]. Colostrum is a thick, yellow substance produced during the first several days postpartum. Healthy mothers produce approximately $80-100 \mathrm{~mL}$ daily. Colostrum is rich in calcium, antibodies, minerals, proteins, potassium, and fat-soluble vitamins. This milk has immunologic qualities that are vital to the infant, and it possesses gastrointestinal properties to facilitate secretion of meconium. Production of colostrum is followed for the next 5-6 days with transitional milk, which provides essential components more closely resembling mature breast milk. Most women will notice a significant change evidenced by the fullness of their breasts and the change in the consistency of the milk. True milk is white and sometimes has a bluish tint. The consistency is similar to that of cow's milk with a sweet taste. Mature breast milk, produced beginning at or near postpartum day 10, produces key components, discussed in the next section.

Numerous factors may affect the supply of breast milk, including anxiety, medications, maternal nutritional status, amount of sleep, exercise, breastfeeding frequency, tactile stimulation, and fluid intake. Breastfeeding mothers should be encouraged to consume generous amounts of fluids and express breast milk every 2-3 hours. The hormonal feedback loop that controls the production and release of prolactin and oxytocin is initiated by suckling or other tactile stimulation of the breast. The greater the amount of suckling or other tactile breast stimulation, the greater the milk supply.

\section{Breast-Feeding}

Because breast-feeding delays the onset of menstruation after pregnancy, a phenomenon that is easily observed, it has often been regarded as a form of birth control [6]. It is, however, only a relatively short-term one. Modern studies in developing countries show that mothers who breastfeed for an extended period do not begin menstruating until an average of ten months after delivery as compared with three months for mothers who do not breast-feed for a long period. It also takes breast-feeding mothers longer to conceive a child after their most recent birth event, perhaps because fertility is not at its height. This sterility is based on the assumption that the infant has little solid food and is entirely dependent on breast-feeding. If solid foods are offered, the window of nonfertility is lessened. Some practices associated with breast-feeding, however, might have lengthened this window. Many peoples including the Greeks and the Romans held that sexual intercourse spoiled the milk and, because some of these same cultures believed that children should be nursed at least for three years, long periods of abstinence would have been associated with breast-feeding. Other factors are involved as well. The onset of menstruation, even with lactating women, is closely associated with levels of nutrition and physical well-being. A comparative study of Bostonian and Taiwanese women who breast-feed indicated that a higher percentage of Boston women had begun to menstruate within six months of weaning than had Taiwanese women. The best advice today for women who are 
breast-feeding and who are also engaging in sexual intercourse is to use one of the methods of contraception available as well.

\section{Abnormalities}

Embryologically, the breasts develop from columns of cells called mammary ridges, which extend along the anterior body wall from the armpits to the upper thighs [2]. Most of the ridges disappear in the course of prenatal development except for the parts in the midthoracic region, which give rise to the breasts and nipples. Sometimes people have extra breasts or nipples. These are most commonly found in the armpits or on the lower chest below and medial to the normal breasts, but they may appear anywhere along the course of the embryonic mammary ridges (the milk line). Extra nipples and breast tissue may be a source of embarrassment to the individual, but usually they do not cause other problems.

Nonproliferative (benign) cystic changes in breast tissue, often called benign cystic disease or benign fibrocystic change (FCC), is a common condition that bears no increased risk for development of cancer. FCC occurs in about one-third of women from the age of twenty to the menopausal period, after which the condition recedes. It is characterized by focal areas of proliferation of glandular and fibrous tissue in the breast associated with localized dilatation of ducts, resulting in the formation of varioussized cysts within the breast. Cystic change appears to be caused by irregularities in the response of the breast tissue to the normal cyclic variations of each menstrual cycle. Clinically, a breast cyst may feel very firm and may appear to be a solid tumor. Ultrasound examination of the breast is often helpful in distinguishing a cystic from a solid mass in the breast. Often, if the physician believes the mass to be a cyst rather than a solid tumor, an attempt is made to aspirate the cyst. A needle is introduced into the breast under local anesthesia. If a cyst is present, the fluid is aspirated and the mass disappears. If no fluid can be obtained, surgical excision is performed.

\section{Pain}

Mastalgia is a symptom complex of breast pain and tenderness, with or without nodularity [7]. Among presenting breast complaints in primary care, mastalgia is at least as common, if not more common, than finding a lump. Most women are concerned about cancer. However, in a study of 987 women whose only complaint was breast pain, $1 \%$ had a malignancy on mammogram. Mastalgia is either cyclic or noncyclic, and the management depends on this categorization. Reassurance, after appropriate evaluation, that the pain is not due to cancer will be sufficient for most women; roughly $15 \%$ will require additional treatment.

Approximately two-thirds of women presenting with breast pain have cyclic mastalgia, which is bilateral pain varying in intensity throughout the menstrual cycle with the premenstrual time often the most painful. It is thought to be hormonally mediated although studies of circulating levels of progesterone, estrogen, prolactin, or quantity of hormone receptors have yielded conflicting results; however, altered hormone receptor sensitivity remains a possibility. The usual age at presentation is 33 to 35 years; the condition also has been reported by postmenopausal women on hormone therapy. Noncyclic mastalgia is usually unilateral, typically occurs in women over the age of 40 years, and is not temporally related to the menstrual cycle.

Postsurgical breast pain may occur at the site of an incision, particularly if the lines of Langer have been crossed. Mondor's disease (phlebitis of the thoracoepigastric vein) may be related to a history of breast surgery, trauma, or radiation. Costochondritis (Tietze syndrome) reportedly accounts for approximately $7 \%$ of noncyclic mastalgia. Ruptured breast implants may also be a cause of localized breast pain. Although subclinical operable breast cancer may present with noncyclic breast pain of recent onset, it is rare that pain is the only presenting symptom in malignancy.

\section{Cancer}

Breast carcinoma occurs in both sexes [2]. It is a rare tumor in men, whose breast tissue is not subjected to stimulation by ovarian hormones, but it is the most frequently diagnosed cancer in women and ranks second as a cause of cancer deaths (exceeded only by lung cancer). Breast cancer incidence rates declined drastically in the early part of the 21st century with the recognition that combined estrogen-progesterone therapy to reduce the symptoms associated with menopause (hormone replacement therapy) was a major risk factor for breast cancer. Additional modifiable risk factors include being overweight, physically inactive, consuming alcohol, or being a heavy smoker. Hormonal factors also influence the risk of breast carcinoma. Women who have never borne children or had their first child after age thirty are at increased risk, as are women who have had early onset of menses (menarche) or late menopause (that is, have had a long menstrual history). High breast tissue density (increased glandular relative to fat tissue) as measured on mammography also may indicate increased risk, but this may be due to the difficulty of early detection in such breasts rather than to heightened risk. There is some tendency for breast carcinoma to run in families, and a woman is at higher than normal risk if her mother or sister has had a breast carcinoma. Inherited mutations can lead to striking increases in breast cancer susceptibility. Two genes (BRCA1 and BRCA2), although rare in the population (less than 1 percent), account for up to 10 percent of all female breast cancer and up to 20 percent of breast cancer that occurs in families. In summary, the etiology of breast cancer is multifactorial and involves genetic background, hormonal status, and poorly defined environmental factors. Significant differences in breast cancer frequencies are found in different populations and socioeconomic groups. Mammography has led to the understanding that breast cancer originates in "in situ disease," which is restricted to the ducts and lobular system and is not (yet) capable of metastasis. Ductal carcinoma in situ (DCIS), the precursor lesion to most breast cancers, is now diagnosed with fivefold greater frequency 
as a result of screening mammography. With time, in situ disease leads to populations of neoplastic cell that can traverse the ductal basement membrane, invade the surrounding tissue, and metastasize to distant sites. Because it is unclear which cases of DCIS will progress to invasive disease, DCIS is treated aggressively with excision and sometimes radiation and hormonal therapy. The process of progression is accompanied by loss of the myoepithelial cell layer surrounding the ducts and lobules of the TDLU.

Substantial evidence supports the use of routine screening mammography; however, recommendations relating to timing and frequency vary by different agencies and countries [8]. About onethird of the abnormalities detected on screening mammograms will be found to be malignant when biopsy is performed. The probability of cancer on a screening mammogram is directly related to the Breast Imaging Reporting and Data System (BIRADS) assessment, and workup should be performed based on this classification. The sensitivity of mammography varies from approximately $60 \%$ to $90 \%$. This sensitivity depends on several factors, including patient age, breast density, tumor size, tumor histology (lobular versus ductal), location, and mammographic appearance. In young women with dense breasts, mammography is less sensitive than in older women with fatty breasts, in whom mammography can detect at least $90 \%$ of malignancies. Smaller tumors, particularly those without calcifications, are more difficult to detect, especially in dense breasts. The lack of sensitivity and the low incidence of breast cancer in young women have led to questions concerning the value of mammography for screening in women 40-50 years of age. The specificity of mammography in women under 50 years varies from about $30 \%$ to $40 \%$ for nonpalpable mammographic abnormalities to $85 \%$ to $90 \%$ for clinically evident malignancies. Currently, the American College of Radiology recommends annual mammography screening for women aged 40 and older and the American Cancer Society recommends screening average-risk women annually starting at the age of 45 and offering mammography to women who choose to do so starting at the age of 40 . Thus, clinicians should have an informed discussion with patients about screening mammography regarding its potential risks (eg, false positives, overdiagnosis) and benefits (eg, early diagnosis), taking into consideration a patient's individual risk factors.

\section{Breast Examination}

Breast self-examination (BSE) was traditionally advocated as a method of self-screening [9]. Over the years, evidence has demonstrated that self-examination does not reduce breast cancer-related mortality and is associated with an increased rate of benign biopsies. Beginning in 2009, the USPSTF (US Preventive Services Task Force) specifically recommended against clinicians teaching the practice of breast self-examination (awarding that service a "D" grade), concluding with a moderate or high certainty that BSE did not have a net benefit for patients.
The new mantra being advocated, in place of the traditional practice of BSE, is the concept of "breast self-awareness," which is being promoted by essentially all organizations, including the ACOG (American Congress of Obstetricians and Gynecologists), ACS (American Cancer Society), and NCCN (National Comprehensive Cancer Network). Rather than a methodically and routinely performed self-exam, this recommendation emphasizes the importance of patients being aware of the way their breasts normally appear and feel. The patient is encouraged to be aware of any change that may occur in their own body and to discuss these changes with their physician. A breast finding brought to a clinician's attention by the patient may be appropriately followed up with either reassurance, clinical breast exam, and/or imaging.

\section{Conclusion}

The first step in breast self-examination is to look for any visible changes in front of the mirror. These can be a change in skin color, a change in the size of a breast, a change in texture, skin indentation, etc. It is also necessary to look at the nipples and see if they have changed shape or color in any way. Most breast changes are benign in nature and patients should not be immediately frightened if they notice changes in the breast. It is necessary to go to the doctor without panic and excessive fear. Without delay.

\section{References}

1. Oyelovo T, Johnson JLB (2018) A Guide to Women's Health. In: $2^{\text {nd }}$ edn), Jones \& Bartlett Learning, LLC, Burlington, USA, pp. 36-37.

2. Reisner EG, Reisner HM (2017) Crowley's an Introduction to Human Disease- Pathology and Pathophysiology Correlations. In: $\left(10^{\text {th }} \mathrm{edn}\right)$, Jones \& Bartlett Learning, LLC, Burlington, USA, pp. 419- 424.

3. Karam A (2013) The Breast in Decherney. In: Nathan AH, Laufer L, Roman AS (Eds.), In: (11 ${ }^{\text {th }}$ edn), Current Diagnosis \& Treatment Obstetrics \& Gynecology. The McGraw-Hill Companies Inc, New York, USA, pp. 249.

4. Ricci SS (2009) Essentials of Maternity, Newborn, and Women's Health Nursing. In: ( $2^{\text {nd }}$ end), Wolters Kluwer Health, Lippincott Williams \& Wilkins, Philadelphia, USA, pp. 52.

5. Conti TD, Patel M, Bhat S (2015) Breastfeeding \& Infant Nutrition. In: South-Paul JE, Matheny SC, Lewis EL (Eds.), In: (4 ${ }^{\text {th }}$ edn), Current Diagnosis \& Treatment in Family Medicine. McGraw-Hill Education, New York, USA, pp. 28-29.

6. Bullough VL (2001) Encyclopedia of Birth Control. ABC-CLIO Inc, Santa Barbara, USA, pp. 38.

7. Schwartz KL (2012) Breast Problems. In: Sloane PD, Slatt LM, Ebell MH, Smith MA, Power DV, Viera AJ (eds.), In: (6 ${ }^{\text {th }}$ edn), Essentials of Family Medicine. Wolters Kluwer, Lippincott Williams \& Wilkins, Philadelphia, USA, pp. 297.

8. Giuliano AE, Hurvitz SA (2018) Breast Disorders. In: Papadakis MA McPhee SJ, Rabow MW (eds.), In: (57 th edn), Current Medical Diagnosis and Treatment 2018. McGraw-Hill Education, New York, USA, pp. 751.

9. Rockoff S, Calandra DJ (2018) Breast Cancer Screening. In: Knaus JV, Jachtorowycz MJ, Adajar AA, Tam T (eds.), Ambulatory Gynecology", Springer Science+Business Media, LLC, Springer Nature, New York, USA, pp. 3. 
(CC) This work is licensed under Creative BY DOI: 10.19080/JGWH.2021.21.556068

\section{Your next submission with Juniper Publishers will reach you the below assets}

- Quality Editorial service

- Swift Peer Review

- Reprints availability

- E-prints Service

- Manuscript Podcast for convenient understanding

- Global attainment for your research

- Manuscript accessibility in different formats

( Pdf, E-pub, Full Tsext, Audio)

- Unceasing customer service

Track the below URL for one-step submission https://juniperpublishers.com/online-submission.php 